\title{
Robert Heim und Emilio Modena (Hrsg.): Jacques Lacan trifft Alfred Lorenzer. Über das Unbewusste und die Sprache, den Trieb und das Begehren (Psychosozial-Verlag, Giessen, 2016) Olaf Knellessen (Zürich)
}

Das vorliegende von Robert Heim und Emilio Modena herausgegebene Buch versammelt die Beiträge einer Tagung, die im Juni 2014 vom Psychoanalytischen Seminar Zürich (PSZ) und der Stiftung für Psychotherapie und Psychoanalyse veranstaltet wurde. Sowohl bei Jacques Lacan als auch bei Alfred Lorenzer steht die Sprache im Zentrum ihrer Theorien und dennoch könnten sie verschiedener kaum sein. «Hob Lorenzer die Freud'sche Lehre des Unbewussten auf die Höhe einer Metatheorie, tauchte Lacan mit seiner «Rückkehr zu Freud in die klassische Psychoanalyse ein, um mit einer vergleichbar anspruchsvollen Theorie das wissenschaftliche und klinische Feld der Psychoanalyse radikal umzupflügen» (S. $129 \mathrm{f}$.), so fasst Robert Heim in seinem abschliessenden, sehr ausgewogenen Beitrag die Differenz, die schon in der Ausrichtung der beiden Theorien besteht.

Es ist also kein einfaches Unterfangen, eine Begegnung zwischen den beiden so verschiedenen Autoren zu Stande zu bringen. Schon das Cover und seine Anordnung der Konterfeis der beiden Protagonisten legt ein Treffen zwischen ihnen nicht unbedingt nahe.

Emilio Modena weist in seiner Einführung darauf hin, dass wir uns als Psychoanalytiker immer wieder eingestehen müssen, «dass wir mit unserem Wissen sehr oft an Grenzen stossen, nicht mehr weiterkommen, verunsichert, ja verzweifelt sind», und ortet die Funktion der Theorie darin, «den Analytiker und die Analytikerin vor Selbstzweifeln zu schützen (...)» (S. 11). Damit markiert er eine Schwierigkeit, an der solche Begegnungen scheitern können. Wenn nämlich Theorien eine solche Funktion haben und die Differenzen zwischen ihnen - wie es für den vorliegenden Fall dem Zitat von Robert Heim schon zu entnehmen ist - sehr grundsätzlich sind, kann dies die Neigung verstärken, sich in Selbstgewissheit zu retten und die Identität mit der eigenen Theorie zu betonen. Eine solche Tendenz ist dem vorliegenden Band anzumerken. So entlarvt gleich zu Beginn PeterWidmer anhand von Descartes und seinem Cogito ergo sum die Identität, die in diesem Ich gefasst werden soll, als Illusion, so betont Ulrike Prokop die Irritation beim 
Verstehen kultureller Erscheinungen als Quintessenz des Vorgehens nach Lorenzer. Bei der Begegnung mit der anderen Theorie und der damit möglicherweise verbundenen Irritation vertraut man allerdings weitgehend doch dem eigenen Wissen und bleibt sich und diesem treu. Von einem Reiz zum Verrat oder abtrünnig zu werden, was ja zu einer Begegnung mit dem Anderen durchaus gehören könnte - wie es die Wahlverwandtschaften, die in diesem Buch einen zentralen Stellenwert haben, vorführen -, ist jedenfalls nicht sehr viel zu spüren.

Didaktik ist da ein sichererWert und die beiden Einführungen von Widmer und Hans-Dieter König in die jeweiligen Theorien vertrauen sich ihm ganz an. Die Faszination am Anderen hält sich da sehr in Grenzen. Trotzdem kommt es zu einem Geplänkel. Aus Empörung über einen Diskussionsbeitrag eines Lacanianers - seine Ausführungen seien eine «Orgie des Verstehens» - ergänzt König seinen Vortrag an der Tagung für dieses Buch um ein weiteres Kapitel, in dem er Lacans Theorie des Spiegelstadiums in der Perspektive von Lorenzers psychoanalytischer Interaktionsund Sozialisationstheorie zu demontieren versucht. Damit wird alles wieder zurechtgerückt. Allerdings bleibt unklar, weshalb die Bemerkung über die Orgie zu einem Vorwurf wurde. Sie könnte auch Zeichen von Irritation und Anziehung durch den Anderen sein, die scheinbar von beiden Theorien als zentrales Moment jeder Begegnung gesucht wird.

Die Mitte des Buches bilden zwei Interpretationen der Wahlverwandtschaften von Goethe. Wahlverwandtschaften vermögen ja - insofern ist das eine schöne Wahl - solcherart Begegnungen sein, um die es in diesem Buch geht.

Marianne Schuller legt auf bestechende Weise einen Ansatz vor, der nicht vom Inhalt, sondern radikal von der Form ausgeht und sich an den Namen der Protagonisten dieses Romans orientiert. Es beginnt mit Eduard, der sich später den Namen Otto gibt. Einen Namen, der sich von vorne und von hinten gleich liest und somit ein Palindrom darstellt, dessen Mitte von einem doppelten $t$ gebildet wird. Der Mittler - der auch das doppelte $t$ aufweist - ist ein Bote, der an Hermes, den Friedensbringer, erinnert, aber einer, der in beide Richtungen wirksam wird, also Beziehungen stiften, sie aber auch auflösen kann. Schon allein dass über die Buchstaben dieses Namens seine Bedeutung entwickelt wird, macht unverkennbar Lacans Ansatz deutlich: «Goethes Roman ist nicht zuletzt deswegen als Sprach-Roman bezeichnet worden, weil er lesbar macht, dass nicht die Figuren diese symbolische Struktur beherrschen, sondern dass sie ihr unterworfen sind: Das ist es, was sie zu Subjekten macht» (S. 72).

Ganz anders ist die Interpretation von Prokop gebaut. Sie geht vom Inhaltlichen - daher ja auch (Tiefen-)Hermeneutik - und vor allem von einer 
Dynamik von Übertragung und Gegenübertragung aus. Bei aller Betonung der Irritation: «Die Tiefenhermeneutik - so Lorenzer - soll solche Unverträglichkeiten im Textgeschehen nicht übergehen» (S. 78), ist diese Dynamik jedoch darauf ausgerichtet, eine Eindeutigkeit, eine geschlossene und auch abgeschlossene Deutung zu geben. Die Brüche, Unverträglichkeiten und Irritationen werden dann - entsprechend zu Lorenzers Verständnis der Symbolfunktion in der Analyse selbst - auf eine Auflösung der Widersprüche ausgerichtet, die zu einer allgemeinverständlichen und -gültigen Deutung führen soll.

In diesem Mittelteil wird in der Methodik etwas von den beträchtlichen Differenzen der beiden Theorieansätze deutlich. Umso bemerkenswerter ist es, dass beide Interpretationen zu einem sehr ähnlichen Schluss kommen. Der heutzutage sich ständig wiederholende Vorbehalt gegenüber einer im Roman beschriebenen «Auflehnung gegen den Tod» (S. 85) geht bei beiden Autorinnen einher mit der «Kritik an der säkularen Welt: an einer Welt, die alles herstellen will, die über alles verfügen will, die Grenzen verleugnet und verschiebt und das Nicht-Verfügbare in der Welt leugnet.» (S. 88). Von hier ist es dann nicht mehr weit zur Anprangerung aktueller totalitärer Glücksversprechen neoliberaler Ökonomie, gentechnischer Manipulationen und dem illusionären Anspruch auf Vollkommenheit auch des Körpers.

Diese inzwischen wenig originelle Position müsste sich den Einwand gefallen lassen, dass die Weiterentwicklung der säkularen Welt solche Auflösung herkömmlicher Beziehungs- und familiärer Strukturen längstens verwirklicht hat, ohne dass es zu einem Zusammenbruch der Kultur, der Gesellschaft oder des Lebens gekommen wäre.

Bei Thierry Simonelli und seiner Arbeit zum «Kleinen Hans» ist es mit den Gemeinsamkeiten schnell wieder zu Ende. Wenn er sich aufWittgensteins Diktum «Wahrscheinlich gibt es viele verschiedene Arten von Träumen und nicht eine Erklärungsweise für alle. Genauso wie es verschiedene Arten von Witzen gibt. Oder genauso wie es verschiedene Arten von Sprache gibt» (S. 107) bezieht, dann jedoch mit dem Hinweis, dass daran jede psychoanalytische Theorie kranke und genau darin der Grund für jenes Diktum von Karl Krauss läge, dass die Psychoanalyse diese «Geisteskrankheit (ist), für deren Therapie sie sich selbst hält.» (S. 106).

André Michels unternimmt dann unter dem Titel Wissen der SpracheSprache des Unbewussten seinerseits den substanziellen Versuch einer Begegnung zwischen Lacan und Lorenzer als Bestimmung ihrer Differenz. In seiner Auseinandersetzung mit Lacan nimmt Lorenzer Bezug auf Lucien Sève, «der Lacans Formulierung (Das Unbewusste ist wie eine Sprache strukturiert einiges abge- 
winnen kann» (S. 121). Sève fragt weiter: «Und warum auf halbem Wege stehen bleiben? Warum dann das sprachliche Verhältnis vom Ensemble der gesellschaftlichen Verhältnisse abstrahieren? Höchstens doch um zu vermeiden, dass man bei den Produktionsverhältnissen anlangt» (S. 123). Damit ist ein wesentlicher Unterschied zwischen Lacan und Lorenzer markiert, insofern es Letzterem eben nicht einfach darum ging, eine Metasprache zu formulieren, so wie es König meinte, sondern vor allem um eine Vermittlung marxistischer, materialistischer Theorie der objektiven Verhältnisse mit der Psychoanalyse als Theorie des Subjekts. Michels schreibt weiter: «Dem Autor geht es vor allem, seinem Verständnis des gesellschaftlichen Materialismus entsprechend, um den Hinweis auf den harten Kern des Realen, auf die 〈Produktionsverhältnisse`, insofern sie «real existierend», d. h. nicht rein sprachlich strukturiert sind» (S. 123).

Damit steht das Verständnis von Produktion im Zentrum der Differenz der beiden Theorien. Wird sie im einen Fall unter nationalökonomischen Aspekten betrachtet, geht es im anderen Fall - unter dem Stichwort von Reproduktion und Übertragung - um ihre triebökonomischen Bedingungen. Beide Perspektiven treffen sich - so könnte man weitergehen - im Fetisch und der von ihm markierten Entfremdung. Wird diese Entfremdung im einen Fall als eine verstanden, die - in Theorie und Praxis - aufgehoben werden kann, ist sie im anderen Fall eine, der nicht zu entkommen ist, die sich - in Reproduktion und Übertragung - immer weiter fortsetzt, ohne den Bruch und die Zäsur aufheben zu können, welche die Sprache und die symbolische Kastration ausmachen. So bleiben Irritation wie auch Anziehung durch das Objekt treibende Kräfte jeder Begegnung.

Sicher etwas vereinfacht kann man sagen, dass die Theorie Lorenzers an eine Überwindung von Entfremdung glaubt, Lacans Denken dem gegenüber skeptischer ist und ein Gelingen vielleicht eher in der Einsicht und Akzeptanz des Scheiterns und des Bruchs sieht. Das hier anvisierte Treffen zwischen Lacan und Lorenzer würde dann eher Lacan Recht geben. Man kann wohl kaum von einer gelungenen Begegnung sprechen. Manchmal schlägt es Funken und blitzen die Unterschiede auf, die Selbstdarstellung und Behauptung des eigenen Wissens gewinnt aber schnell wieder Überhand.

In diesem Sinn stellt Robert Heim in seinem dieses Buch abschliessenden Encore solche Begegnungen von Theorien und ihren Begründern unter das Zeichen der Liebe, indem er die Liebe zur Theorie als Aufleben der Übertragungsliebe versteht. Sie ist im konkreten Fall derWeitergabe psychoanalytischen Wissens idealtypisch «vom Modell der Beziehung zwischen einem Meister und einem Schüler (...) getragen» (S. 166) und kreist um die Anerkennung und Zustimmung des Anderen 
und das Bemühen, ihm zu entsprechen und ihm gleich zu werden. Übertragung ist aber, das ist unsere Erfahrung, nicht nur eine der Liebe, sondern auch eine des Triebs und seines Konflikts.

Begegnungen - auch zwischen Theorien - sind also kein einfaches Unterfangen. Sie stehen im Zeichen des Scheiterns ebenso wie in der Hoffnung auf Gelingen. Und das - so könnte man sagen - wird in diesem Buch eindrücklich vorgeführt. Es lohnt sich, dieses Buch gerade unter diesem Aspekt zu lesen. Dann wird man sehen, wo einem das Herz schlägt. 